\title{
reviews
}

OVER the past decade there has appeared a number of books devoted to the subject of exobiology in general, and extraterrestrial technological communities in particular. The subject is exquisitely fascinating, provocative, and distinguished by a current lack of concrete observational data. These ingredients form a fertile situation for all sorts of unsound speculation so that it is particularly important to have available books on the subject written by competent scientists with established reputations.

One such individual is Ronald Bracewell, of Stanford University, who was among the first contributors to the emergence of scientific consideration of extraterrestrial 'intelligent' communities and the problems and possibilities of radio communication with them. In what Bracewell clearly regards as the pioneering work on the subject, Giuseppi Cocconi and Phillip Morrison suggested in 1959 that radio signals from a technical community in outer space might be directed at us at $1,420 \mathrm{MHz}$, a natural choice of frequency corresponding to the emission from hydrogen.

Following this proposal, Bracewell detailed three distinct cases for consideration: abundant life, with the nearest community within 30 light years; sparse life within say 30-300 light years; and rare life (beyond 300 light years). He pointed out an important connection between the distance of the nearest community, and the average lifetime of the community. If this lifetime is to be measured in many millions of years, then life in the galaxy may well be abundant.

These arguments, together with some technical considerations about radio

\section{Intelligent life in space}

\author{
P. C. W. Davies
}

The Galactic Club: Intelligent Life in Outer Space. By Ronald N. Bracewell. Pp. 141. (Freeman: San Francisco and Reading, 1975.) Cloth £3.70; Paper $£ 1.90$.

telescopes and search patterns, are presented in a short volume called The Galactic Club, a term which refers to the expected network of information exchange between galactic communities. This is a well written and interesting book, with a rather unusual presentation. Many full page paintings and lithographs of a distinctive style punctuate the text, though their connection with the subject matter is occasionally obscure.

The book encompasses a rather odd conjunction of topics. After a brief introduction to the astronomical, chemical and biological basis of exobiology, Bracewell resurrects the Velikovsky affair recalling that author's fantastic speculations about Solar System cataclysms. Returning to the main theme, some discussion is presented on the exact procedure whereby mankind might intercept interstellar messages, the nature of the message and the response. A chapter is devoted to the ambitious and awesome scheme known as Project Cyclops which proposes a vast array of radio telescopes all busily skimming the sky for messages.

Another chapter is devoted to the remarkable idea of interstellar messenger probes. Based on the assumption that near-luminal space probes are unlikely to be a practicable possibility at any level of technological attainment, it is suggested that, rather than indulge in interstellar travel themselves, intelligent space people would send out small probes at modest speed, to lie in wait around likely looking stars in the hope of eventually contacting a nascent technical community. The strategy adopted by such an interloper would be to echo domestic radio messages, thereby attracting the attention of an assured audience. The idea is bound to provoke the thought: is there such a probe in the region of the Earth even now?

This type of discussion leads quite naturally to the question of whether the Earth has been (or still is) the recipient of extraterrestrial visitation. In this context, some of the highly popular and evidently fanciful claims of Erich Von Daniken are briefly mentioned, with the conclusion that they satisfy a deep sociological need and "represent a substitution of faith for reason".

Further spectacles are provided by an outline of Gerard O'Neill's amazing studies of spinning space cylinders. These monster stations, envisaged as many miles across, are one idea of a future space colony.

The Galactic Club will have a considerable appeal to popular readership, providing as it does an intellectual journey into a strange and wonderful world of cosmic speculation.

To the scientific community it presents an interesting background to astronomy and space science and, at a more fundamental level, serves to remind us all of a wider perspective of human society.
DISPERSION relations have been a part of the armoury of high energy physics for nearly 20 years now. It may seem surprising that not very many monographs have been exclusively devoted to this subject over the years. I believe that even a casual reading of this latest offering from Drs Queen and Violini will provide a clue as to why that is; on its own the subject can be rather uninspiring, and the authors have not succeeded in showing otherwise. Indeed, the text is written in such a dry, sparse manner with little or no indication of the excitement inherent in fundamental particle physics that I would never dare recommend it as a first introduction to my graduate students. Having said this, I must say that the book is well organ-

\section{Dry dispersion}

\section{P. G. Williams}

Dispersion Theory in High-Energy Physics. By N. M. Queen and G. Violini. Pp. $x i+202$. (Macmillan: London and Basingstoke, January 1975.) £12.00.

ised, very carefully written and contains a useful combination of material not previously available in book form.

After a short introduction to scattering formalism the authors obtain dispersion relations from causality, studiously avoiding any complications. Then follow two chapters, one on the Mandelstam representation, the other a rather nice treatment of high energy bounds. Thereafter, we find a six- chapter catalogue of various types of dispersion relations (excluding partial wave dispersion relations by explicit choice) and their applications. This is relieved by a chapter on Regge theory. At least I had hoped it would be until I read it and found the subject dried out to its very bones by its rather formal treatment. The final chapter, on the analytic continuation techniques of Cutkosky and Deo, is rather useful.

I can't say I welcome this new addition to the literature : the essential parts of the book are better suited to a review article; sections added by way of introduction for students are formal and not at all well physically motivated. And it is all at the astronomic price of $£ 12.00$ for a mere 200 pages. 